\title{
Thermal Model Parameter Estimation for HVAC Facility using Recursive Least Square Method
}

\author{
Dae Ki Kim ${ }^{1,}$, Kyu Chul Lee ${ }^{1, b}$, Sung Hyun Yoo, c, Jung Hoon Kim, \\ Choon Ki Ahn ${ }^{1, e, *}$, Myotaeg Lim ${ }^{1, f}$ \\ ${ }^{1}$ School of Electrical Engineering, Korea University, Seoul, Republic of Korea \\ acroura@korea.ac.kr, ${ }^{b} \mathrm{lkc88@korea.ac.kr,} \mathrm{cysh88@korea.ac.kr,}$

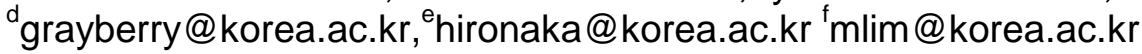

Keywords: Heating, Ventilating and Air Conditioning (HVAC), Home Energy Management System (HEMS), Parameter estimation, Recursive least square.

\begin{abstract}
In this paper, the parameter estimation of thermal dynamic discrete-time system for heating, ventilating and air conditioning (HVAC) is studied using recursive least square method. Parameters are estimated by using recursive least square method. Thermodynamic model of indoor test bed is formulated by outdoor temperature, HVAC power consumption, and thermal noise and estimated thermal dynamic parameters show improved performance of thermodynamic model for day-ahead indoor temperature compared to original least square parameter estimation approach. In this research, the experiment is implemented to verify the performance of proposed approach in a laboratory environment.
\end{abstract}

\section{Introduction}

The researches on peak-time load reduction and price volatility have been studied. It is because peak-time load has great impact on energy saving problem. Especially, many researches on home energy management problem have been highly focused on [1-5]. Appliances in household are classified as two categories including interruptible device and delayable device. Delayable devices are shifted from highly used time interval called peak-time to other time interval. To evaluate proper time interval to be moved, cost function is applied considering power consumption and user comfort. Through this process, both reduction of electricity cost and sub optimal comfortability can be obtained.

During last decades, many researchers have focused on the problem of heating, ventilation, air conditioning (HVAC) [6-8]. Since HVAC facilities have great part of load on peak-time, the problem of HVAC load reduction is important research issue. Furthermore, since HVAC is used to regulate indoor temperature of household and this information is applied to obtain future horizon data used by various controllers including model predictive controller (MPC), an accurate thermal dynamic model formulation is important. This fact motivated many researchers to formulate various type of thermal dynamic models [9-10]. However, it is noted that although thermal dynamic model predicts future indoor temperature well, temperature pattern changes its own dynamic daily property. To deal with this issue, authors in [11] proposed least square (LS) method to estimate thermodynamic model parameters. LS method showed that it has simple and fast properties but it is very sensitive to outliers, which are thermal noises in case of thermal dynamic model. To solve this problem, we propose recursive least square (RLS) method to obtain thermal dynamic model parameters. Since RLS method is less sensitive to outliers, this can be a good candidate for obtaining thermal model parameters. To the authors best knowledge, there have been yet no results obtained on RLS method to obtain thermal dynamic model parameters and this motivated us to investigate this research.

The remainder of this paper is organized as follows. In the next section, the thermodynamic discrete model and recursive least square method are intraduced. Numerical experiments are 
presented in Experimental Results. Finally conclusion is summarized.

\section{Parameter of Thermodynamic Model}

Thermodynamic Model of Zone Temperature for HVAC. In this section, we introduce discrete-time thermodynamic equation including HVAC unit. The resistive-capacitive (RC) network model is commonly used to estimate zone temperature. This paper estimate zone temperature with modified RC network model which includes thermal load effect [12]. Consider the following form:

$$
T_{z}[k+1]=T_{z}[k]+a\left(T_{a}[k]-T_{z}[k]\right)+b P_{A C}[k]+c\left(d-T_{z}[k]\right),
$$

where, ambient energy flow parameter $a$, air conditioner energy flow parameter $b$, thermal load energy flow parameter $c$ and thermal load temperature $d, T_{z}[k]$ is zone temperature, $T_{a}[k]$ is ambient temperature, and $P_{A C}[k]$ is air conditioner power consumption. The zone temperature state-space form of the discrete dynamic thermodynamic model (1) can be formulated as follows:

$$
\begin{aligned}
x[k+1] & =A x[k]+B u[k], \\
y[k] & =C x[k]+D u[k],
\end{aligned}
$$

where,

$$
\begin{aligned}
& A=[1-a-c], \quad B=\left[\begin{array}{lll}
a & b & c d
\end{array}\right], \quad C=1, \quad D=0, \\
& x[k]=T_{z}[k], \text { and } u(k)=\left[\begin{array}{lll}
T_{a}[k] & P_{A C}[k] & 1
\end{array}\right]^{T} .
\end{aligned}
$$

Recursive Least Square Method. We can sequentially obtain real measurement data with Home Energy Management System (HEMS). RLS method generates more accurate results with less computational effort than LS method. Even if thermal feature of zone is changed, RLS method can adjust parameters fast and easily. RLS method for updating parameter is represented as follows:

$$
\begin{aligned}
& K[k]=P[k-1] H^{T}[k]\left(H[k] P[k-1] H^{T}[k]+R[k]\right)^{-1}, \\
& \hat{X}[k]=\hat{X}[k-1]+K[k](Y[k]-H[k] \hat{X}[k-1]), \\
& P[k]=(I-K[k] H[k]) P[k-1](I-K[k] H[k])^{T}+K[k] R[k] K^{T}[k],
\end{aligned}
$$

where,

$$
X[k]=[A[k] \quad B[k]]^{T}, Y[k]=T_{z}[k], H[k]=\left[\begin{array}{lll}
T_{z}[k] & T_{a}[k] \quad P_{A C}[k] & 1
\end{array}\right],
$$

$\mathrm{R}[\mathrm{k}]$ is measurement covariance matrix, $\mathrm{P}[\mathrm{k}]$ is estimation error covariance, and $\mathrm{K}[\mathrm{k}]$ is minimizing factor of the cost function.

\section{Experimental Test Bed}

The thermodynamic model data was obtained by experiment at \#511B in the College of Science \& Engineering at Korea University, Seoul, Korea. The experiment was carried out from thermodynamic test bed with its dimension shown in Fig. 1. Test bed temperature of the room was collected by two thermometers. One thermometer was installed in the middle of room to measure test bed zone temperature. Another thermometer was positioned at the outside of window to measure ambient temperature. As thermometer, Thermometer (e.g. Labjack Digit-TLH data logger) has operating range from $-35^{\circ} \mathrm{C}$ to $85^{\circ} \mathrm{C}$ and $0.3^{\circ} \mathrm{C}$ measurement error. The test bed consists of one air conditioner, two windows, and 3 computers as heat source. Zone information of test bed is area of $35 \mathrm{~m}^{2}$ and volume of 
$81.2 \mathrm{~m}^{3}$, and window size of $3.4 \mathrm{~m}^{2}$. We found thermodynamic parameter experimentally with the measurement data of this test bed.

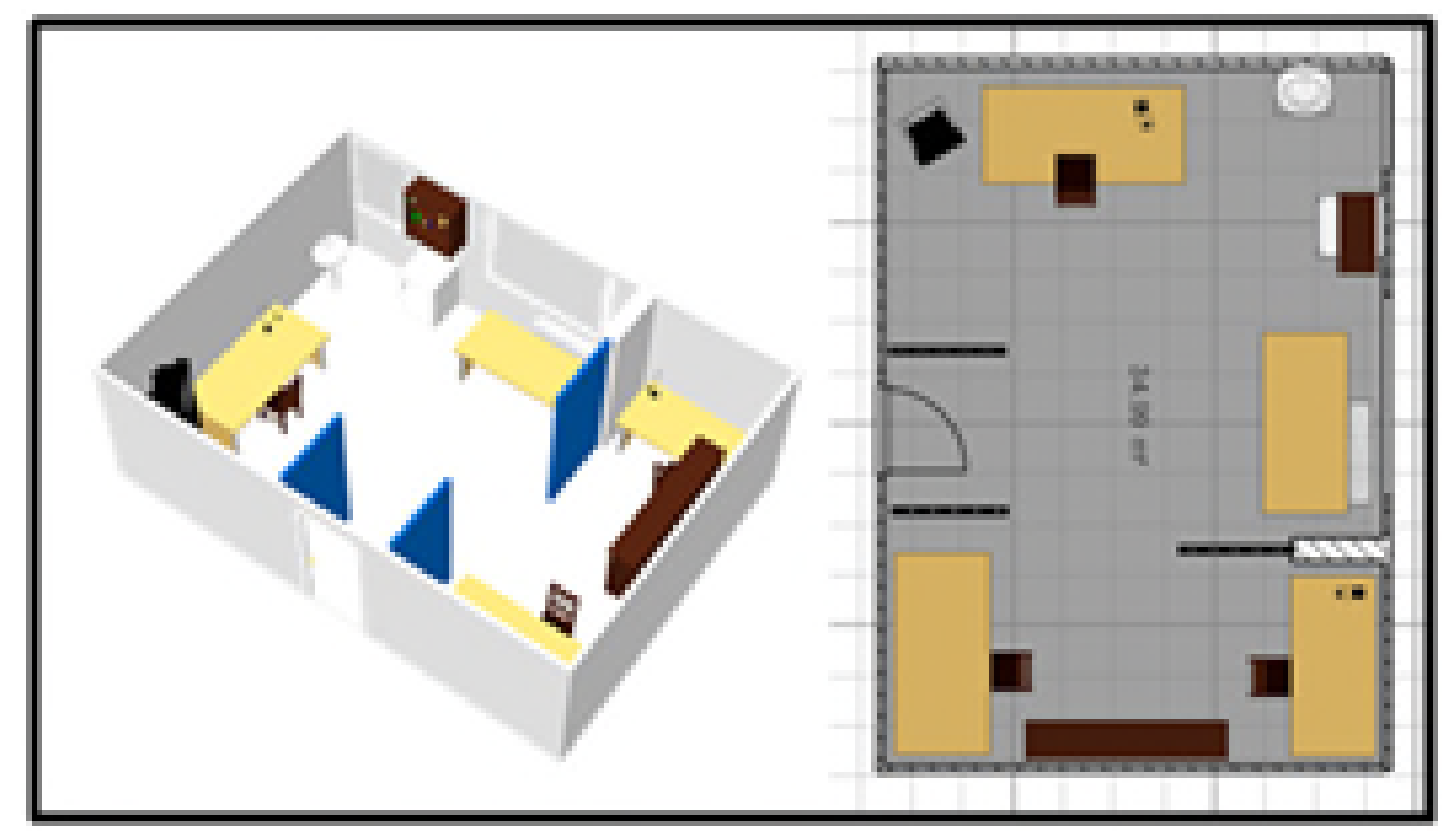

Fig. 1 Test bed layout.

\section{Experimental Results}

In this section, the estimated parameters set and prediction error are presented. We collected data within a month period from July 1, 2014 to July 31, 2014. We experiment to find new thermodynamic parameters when exist change of thermal feature. LS method parameters and RLS method parameters are listed in Table 1.

Table 1. Parameters.

\begin{tabular}{|c|c|c|}
\hline Heading level & LS parameter & RLS parameter \\
\hline$a$ & 0.1404 & 0.1142 \\
\hline$b$ & -1.1105 & -0.8160 \\
\hline$c$ & 27.973 & 27.258 \\
\hline$d$ & 0.4382 & 0.4444 \\
\hline
\end{tabular}

Since unit time of the discrete thermodynamic equation is 10 minute, recursive least square method can learn 144 times per day. Fig. 2 shows how parameters are adjusted to sequentially. Parameters converge into final value after 490 learning time within $6 \%$ error rate. Fig. 3 shows real zone temperature and estimated temperature by using both methods. Proper tendency is founded within RLS method estimation, LS method estimation, and the measured zone temperature data. But it is clear that the estimated data, which uses RLS method, has better performance than LS method. Fig. 4 shows accuracy of RLS method more clearly. Error tendency of RLS method is similar to LS method. But absolute values of RLS method error are obviously smaller than LS method. Table 2 presents the mean squared prediction error value of each method. Mean squared prediction error of RLS method is less than $51.77 \%$ compared to LS method. 


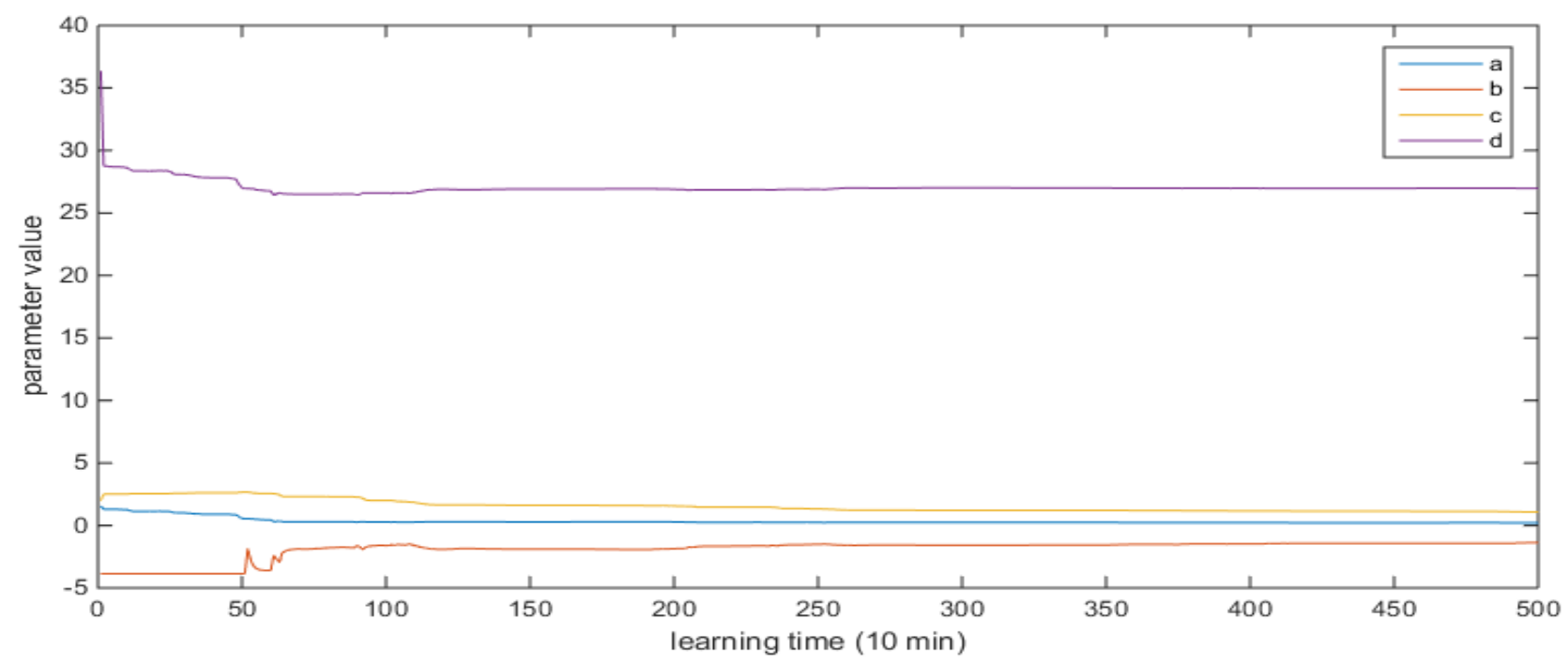

Fig. 2 Adjusting parameters.

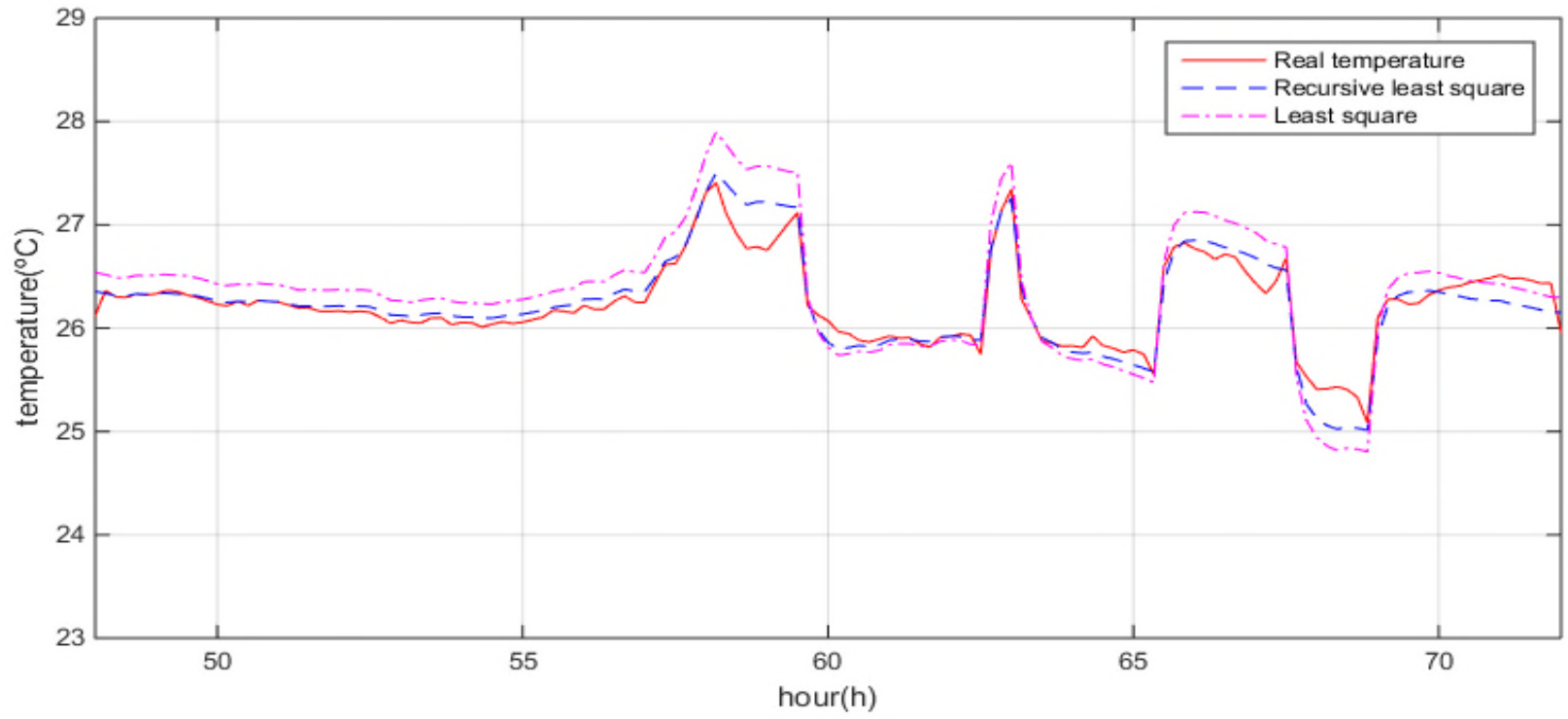

Fig. 3 Estimated zone temperatures.

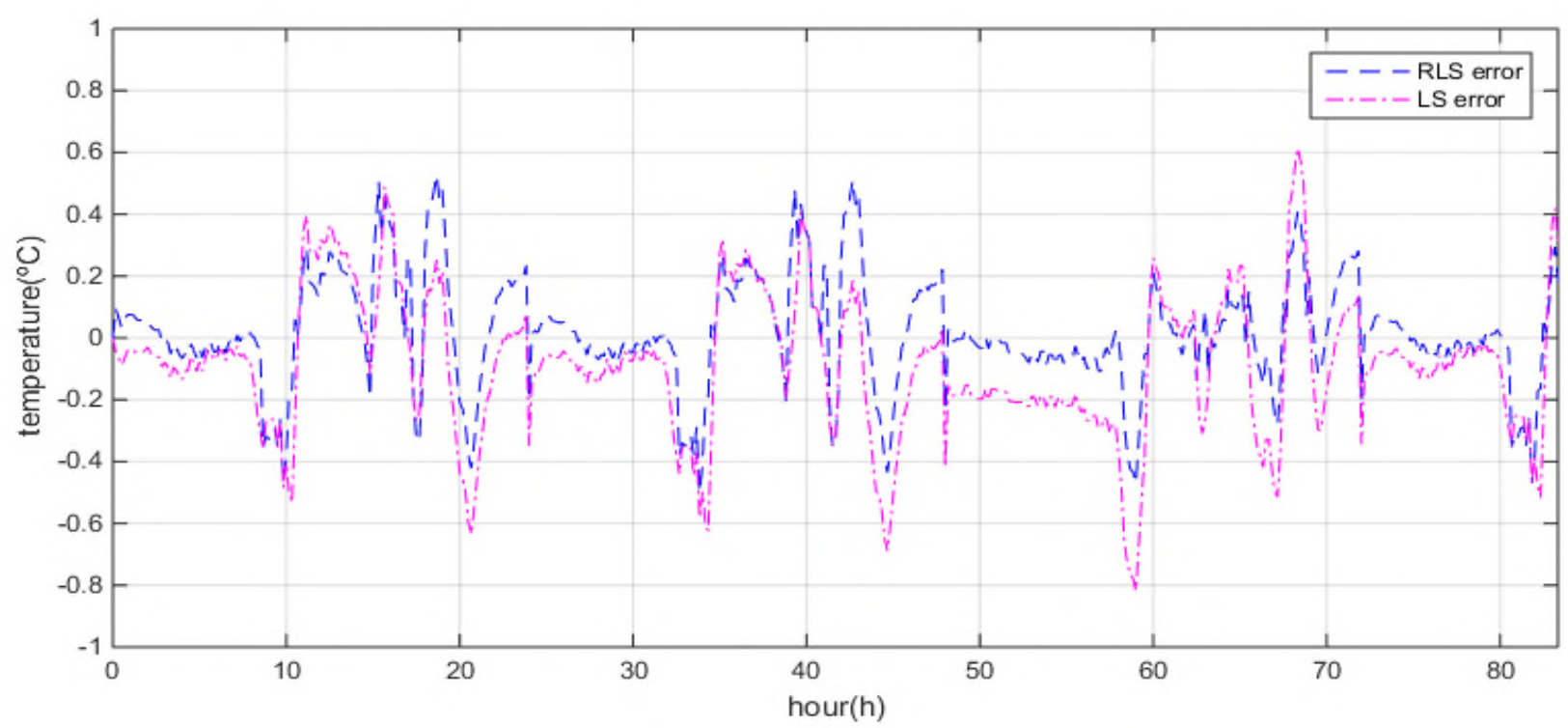

Fig. 4 Estimation error. 
Table 2. Mean squared prediction error.

\begin{tabular}{|c|c|c|}
\hline & LS method & RLS method \\
\hline Mean squared prediction error & 0.0564 & 0.0272 \\
\hline
\end{tabular}

\section{Conclusion}

We investigated the parameter estimation of thermal dynamic discrete-time system for heating, ventilating and air conditioning (HVAC) using recursive least square method in the laboratory environment. The thermodynamic model of zone temperature using proposed estimated parameter showed less mean square prediction error for tracking real zone temperature compared to least square approach. In our future work, we will apply this result to home energy management system to enhance household energy efficiency for home appliances including interruptible device and delayable device.

\section{Acknowledgement}

This work was supported by "Human Resources program in Energy Technology" of the Korea Institute of Energy Technology Evaluation and Planning(KETEP) granted financial resource from the Ministry of Trade, Industry \& Energy, Requblic of Korea(No. 20154030200610)

\section{References}

[1] A. J. Conejo, J. M. Morales, L. Baringo, Real-Time Demand Response Model. IEEE Trans. Smart Grid, 1(3) (2010) 236-242.

[2] Z. Chen, L. Wu, Y. Fu, Real-Time Price-Based Demand Response Management for Residential Appliances via Stochastic Optimization and Robust Optimization. IEEE Trans. Smart Grid, 3(4) (2012) 1822-1831.

[3] T. Logenthiran, D. Srinivasan, T. Z. Shun, Demand Side Management in Smart Grid Using Heuristic Optimization. IEEE Trans. Smart Grid, 3(3) (2012) 1244-1252.

[4] M. Parvania, M. Fotuhi-Firuzabad, M. Shahidehpour: Optimal Demand Response Aggregation in Wholesale Electricity Markets. IEEE Trans. Smart Grid, 4(4) (2013) 1957-1965.

[5] A. H. Mohsenian-Rad, A. Leon-Garcia, Optimal Residential Load Control with Price Prediction in Real-Time Electricity Pricing Environments. IEEE Trans. Smart Grid, 1(2) (2010) 120-133.

[6] Y. Y. Hong, J. K. Lin, C. P. Wu, C. C. Chuang, Multi-Objective Air-Conditioning Control Considering Fuzzy Parameters Using Immune Clonal Selection Programming. IEEE Trans. Smart Grid, 3(4) (2012) 1603-1610.

[7] A. Arabali, M. Ghofrani, M. Etezadi-Amoli, M. S. Fadali, Y. Baghzouz, Genetic-Algorithm-Based Optimization Approach for Energy Management. IEEE Trans. Smart Grid, 4(1) (2013) 162-170.

[8] Y. D. Ma, F. Borrelli, B. Hencey, B. Coffey, S. Bengea, P. Haves, Model Predictive Control for the Operation of Building Cooling Systems. IEEE Trans. Contr. Syst. Technol. 20(3) (2012) 796-803.

[9] C. Chen, J. H. Wang, Y. S. Heo, S. Kishore, MPC-Based Appliance Scheduling for Residential Building Energy Management Controller. IEEE Trans. Smart Grid, 4(3) (2013) 1401-1410.

[10] H. Huang, L. Chen, E. Hu, Model Predictive Control for Energy-Efficient Buildings: An Airport Terminal Building Study. 1th IEEE International Conference on Control \& Automation (ICCA) (2014).

[11] H. D. Choi, S. W. Rhee, C. K. Ahn, M. T. Lim, AC Scheduling Based on Thermodynamics of Indoor for On-Campus Small Data. J. Power Energ. Eng. 3(4) (2015) 282-288. 